\title{
LA-6897-MS
}

Informal Report

UC-32

Issued: July 1977

\section{Two-Dimensional Maximum Entropy Image Restoration}

\author{
J. E. Brolley \\ R. B. Lazarus \\ B. R. Suydam \\ H. J. Trussell
}

\section{scientific laboratory}

of the University of California

lOS ALAMOS, NEW MEXICO 87545

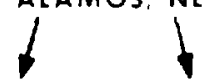

An Affirmative Action / Equal Opportunity Employer 
TWO-DIMENSIONAL MAXIMUM ENTROPY IMAGE RESTORATION

by
J. E. Brolley
R. B. Lazarus
B. R. Suydam
H. J. Trussel1

\section{ABSTRACT}

An optical check problem was constructed to test $P$ LOG $P$ maximum entropy restoration of an extremely distorted image. Useful recovery of the original image was obtained. Comparison with maximun a posteriori restoration is made.

\section{INTRODUCTION}

No image restoration techniques except the $P$ LOG $P$ form of maximum entropy analysis appear to offer the possibility of super resolution. For the nonce we mean this to imply that the restored object space contains mo:e pixels than the original image space, and both spaces have the same boundaries. We further imply that the Rayleigh resolution limit may be exceeded. P LOG P is a label which identifies the form of entropy measure that is used. Analysis using the LOG $P$ form was introduced by Burg. ${ }^{1}$ The other form was introduced by Frieden. $^{2}$

We have developed the two-dimensional P LOG $P$ analysis and applied it to resioring a pinhole image produced by $2-k e V X$ rays. The physical principles and an outline of the mathematical procedure we employed have been reported. ${ }^{3}$ Prior to attacking the $X$-ray image problem we had tested the method on simple synthetic examples with various amounts of noise mixed in. Results from these simple examples indicated it was reasonable to execute the $X$-ray problem. However, it became apparent that numerous significant potential applications existed and it was therefore desirable to consider a reasonably difificult 
problem for which we knew the answer. We have, therefore, chosen to essentially repeat the earlier pinhole calculation for a controlled set of data.

\section{APPARATUS}

B. Brixner and M. Winkler have kindly set up a pinhole camera system for operation in the visible spectrum. The green line of mercury, $5461 \AA$, was used. Apart from the wavelengths involved the present problem differs markedly from the $x$-ray case in that the new pinhole is relatively much larger than in the $x$-ray case. One consequence of this is that the image will be severely distorted and, in fact, hardly resembles the object. Another consequence is that diffraction formalism is different.

In Fig. 1 we note the schematic form of the pinhole camera. A pattern resembling a pinwheel was placed on film and illuminated with monochromatic light. The pinwheel thus appeared as a glowing object in a dark field. A PDS raster scan of the target film was performed by R. Bagley and is shown in Fig. 2.

\section{TEST GEOMETRY}

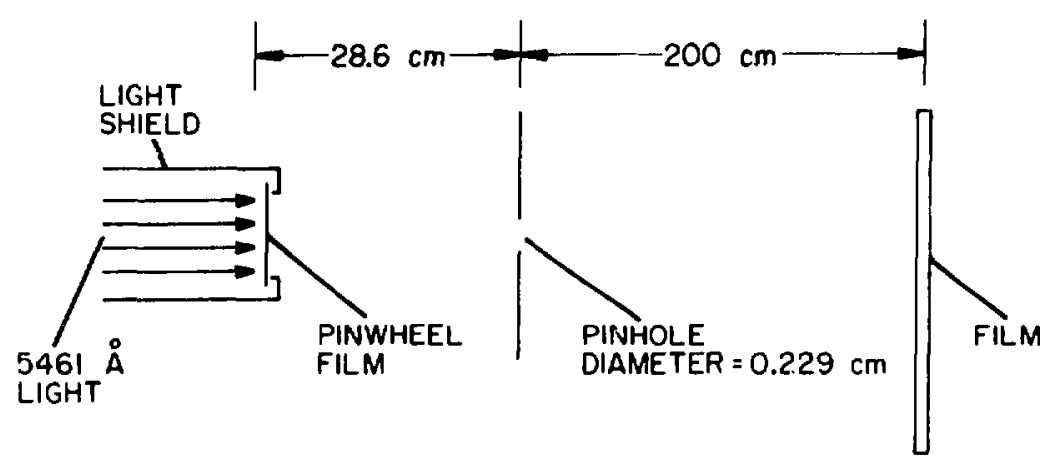

Fig. 1.

Schematic form of the pinhole camera. 
Optical considerations indicate that the pointspread function for the present problem can be adequately inferred from geometrical optics. However, it was deemed instructive to calculate it from Fresnel diffraction theory as we did in the $X$-ray case. The approximation previously used is no longer valid. Instead the real and imagiary parts of the electric field amplitudes are given by

$$
E_{j}(r, Z)=\int_{0}^{a} J_{0}\left(\frac{k r \bar{r}}{Z}\right) \cos \left(\frac{k \alpha \bar{r}^{2}}{2 Z}\right) \bar{r} d \bar{r}
$$

and

$$
E_{2}(r, Z)=\int_{0}^{a} J_{0}\left(\frac{k r \bar{r}}{Z}\right) \sin \left(\frac{k \alpha \bar{r}^{2}}{2 Z}\right) \bar{r} d \bar{r},
$$

where

$$
\begin{aligned}
& k=2 \pi / \lambda, \\
& x=1+Z / R, \\
& Z=\text { distance from pinhole to plane, } \\
& R=\text { distance from point source to pinhole, } \\
& a=\text { radius of the pinhole, } \\
& r=\text { radius from the geometrical center of the pattern in the image plane, } \\
& \lambda=\text { photon wavelength. }
\end{aligned}
$$

The intensity is then

$$
I-E_{1}^{2}+E_{2}^{2}
$$

I is the power density on the image film. In Fig. 3, I is plotted as a function of radius on the image film. The pattern is reminiscent of that produced by the parallel jaw slit. Also plotted in Fig. 3 is the integrated power contained in a circle as a function of radius. As expected, essentially all of the power lies within the geometrically projected radius of the pinhole. Our computation of the pointspread function utilized a smoothed version of $I(r)$.

It is clear that pointspread function will cause extreme smearing of the image because of its large $95 \%$ power point. This is confirmed by the PDS image 


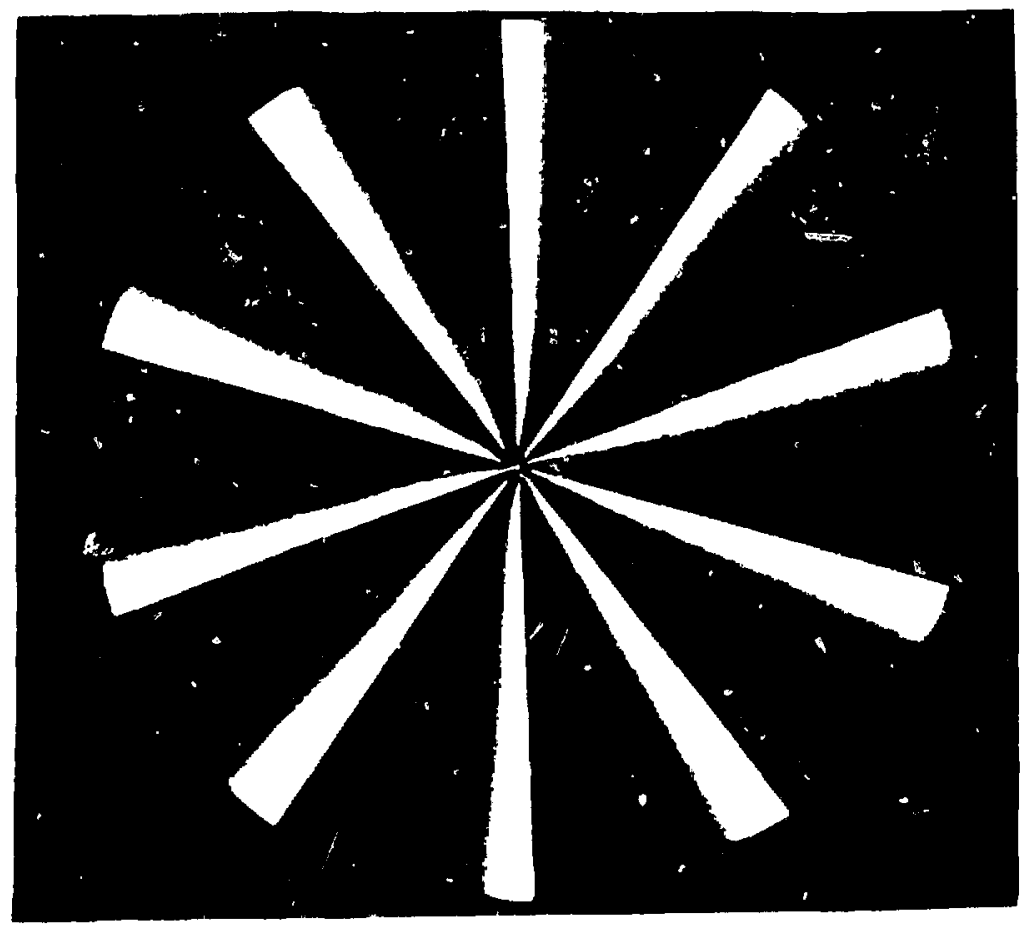

Fig. 2.

COMTAL presentation of the cijject film pattern made from a PDS scan.

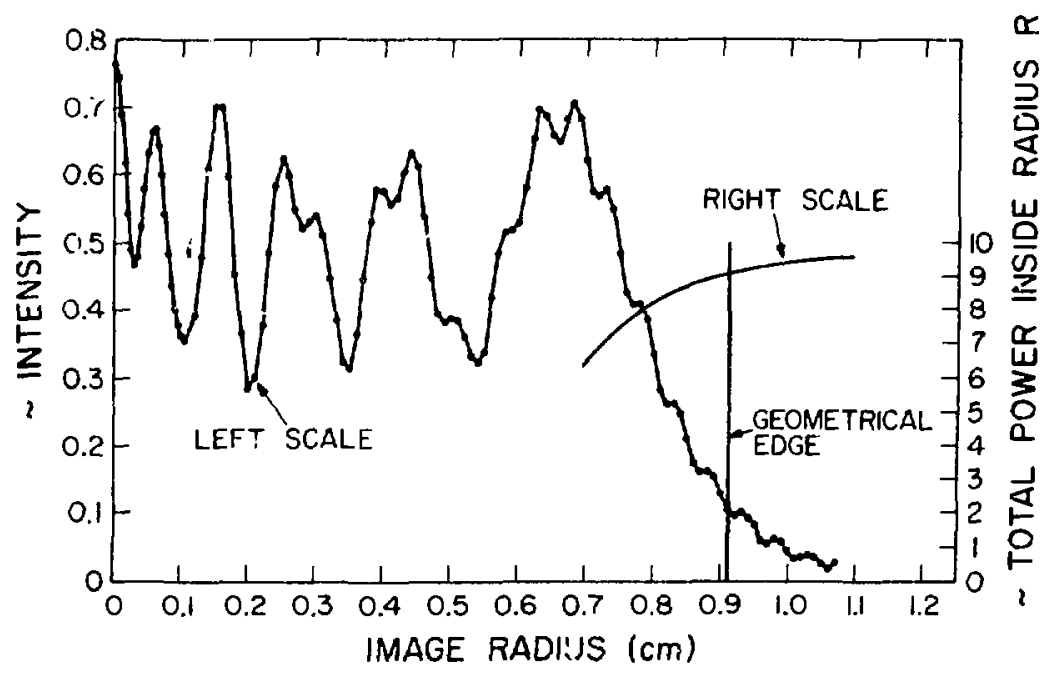

Fig. 3.

Radial dependence of the pointspread function in the image plane (left axis). Radial dependence of the power flowing through a circle of radius $r$ (right axis). 
shown in Fig. 4. It will be noted that not only is the taper of the spokes lost, but a large complicated pattern occurs in the center. The 1024 by 1024 pixel picture shown in Fig. 4 was processed further before being used as input data for the maximum entropy calculation. The film densities were exponentiated to simulate photon power, then an average background was estimated from a region where there was no image. This was subtracted from the power matrix of the picture. Some small negative numbers then occurred in the resultant power matrix. Our method of aralysis takes cognizance of the negative numbers. For reasons of computational complexity we further reduce the number of pixels by averaging to a final 64 by 64 pixel image. This results in still further smearing as may be noted in Fig. 5 . The image in Fig. 5 shows the film densities as recorded on the negative. Figs. 3 and 4 have had the densities reversed to show a positive image.

\section{RESULTS}

We have performed the maximum entropy analysis for a restored image space of 80 by 80 pixels. In this restoration $\rho=150$ was used. In Ref. 3 it is noted that o measures the relative weights of the signal and noise entropies, and that increasing $\rho$ usually leads to sharper images. The result is shown in Fig. 6 .

The linear maximum a posteriori (MAP) restoration scheme ${ }^{4}$ was chosen to compare with the maximum entropy method. This method uses a minimum of a priori information to produce good results. In the case of very low noise levels, which is the case in this example, the linear MAP filter approaches the inverse filter. This is a characteristic of most linear filters and thus tends to lessen the importance of which filter is chosen for the comparison.

The restoration produced by linear MAP is shown in Fig. 7. This figure is printed as a negative as Fig. 5. As with any linear filter the output can, and in this case does, have negative values. This is the effect against which the maximum entropy method guards. The result of the linear filter in Fig. 7 shows sharp edges but at the price of noticeable ringing near the outside. Computation time was 20 seconds on the CDC 7600 . 


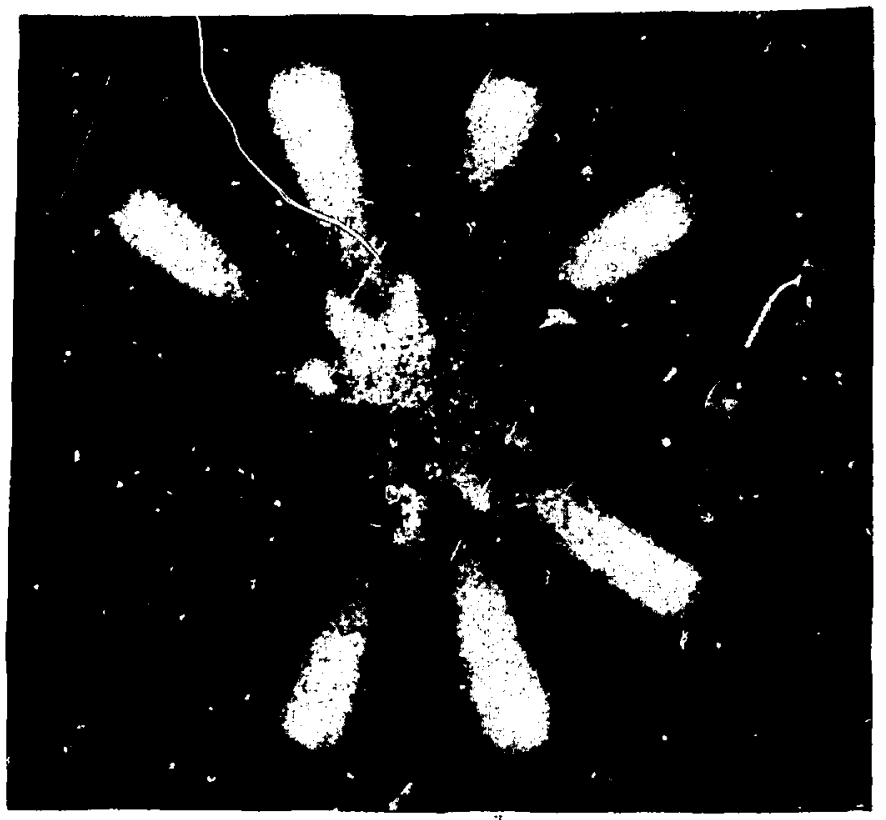

Fig. 4.

COMTAL presentation of the image film made from a PDS scan, 1024 by 1024 pixels.

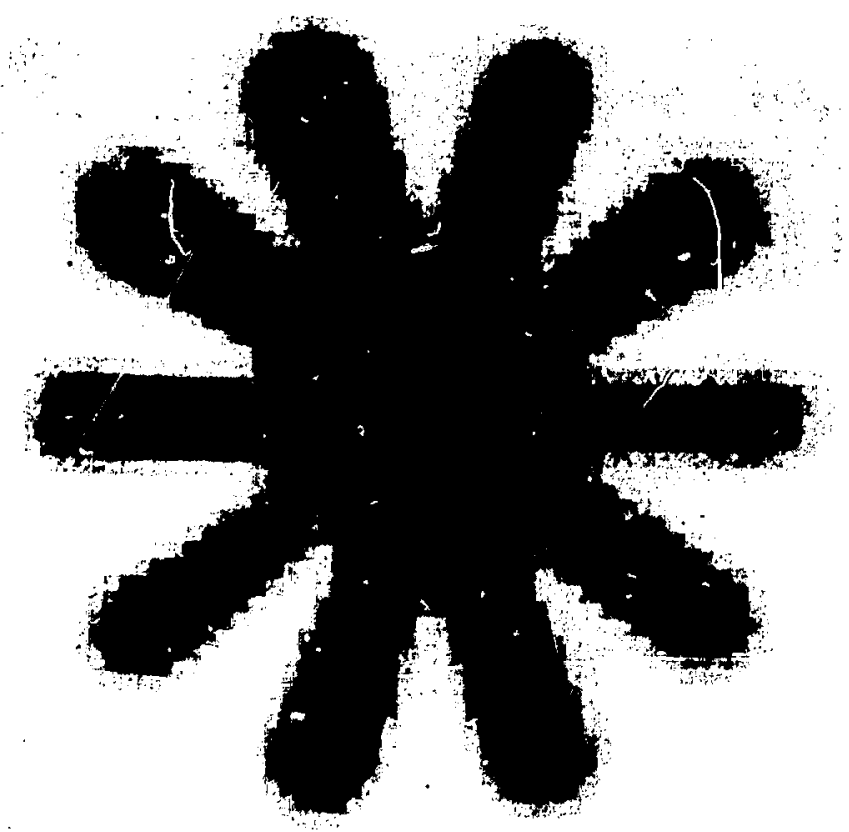

Fig. 5.

COMTAL presentation of the compressed image, 64 by 64 pixels. 


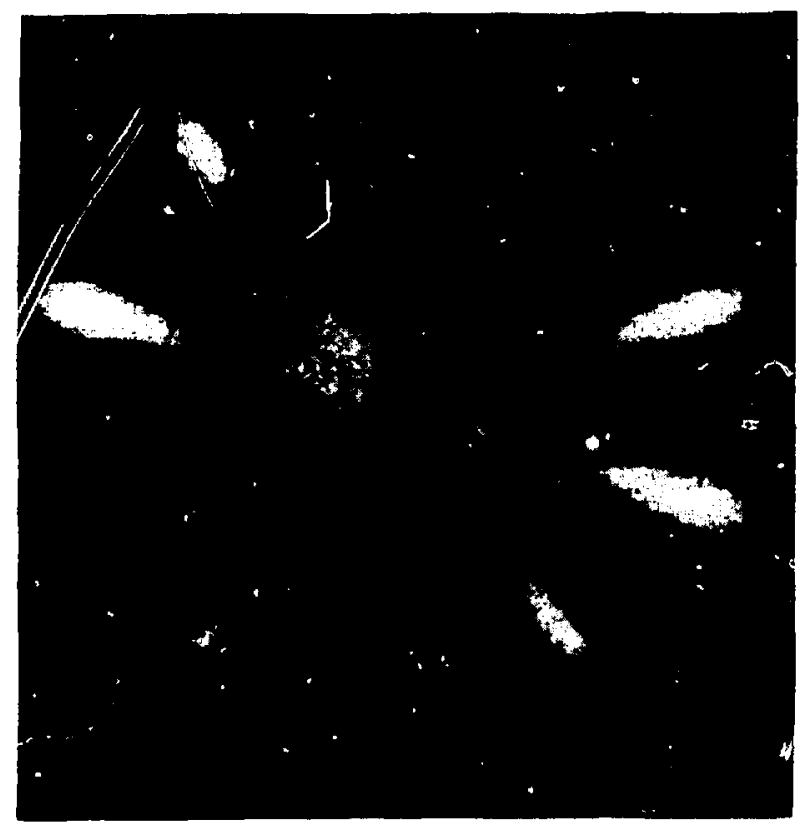

Fig. 6 .

COMTAL presentation of the restored object, 80 by 80 pixels.

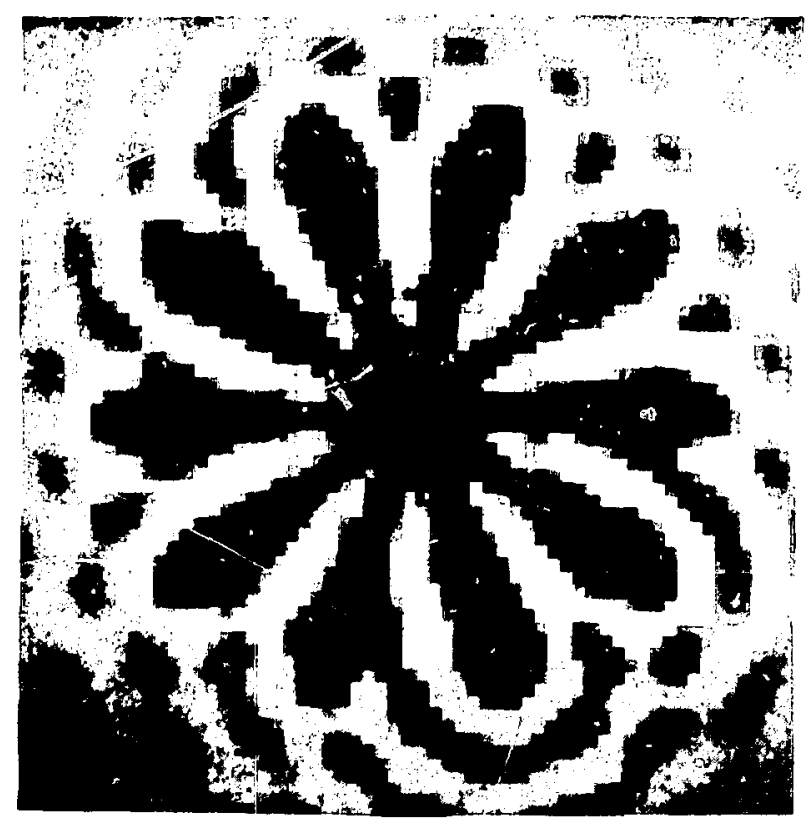

Fig. 7.

COMTAL presentation of the maximum a posteriori restoration, 64 by 64 pixels. 
IV. CONCLUSIONS

The present analysis has recovered the taper of the pinwheel spokes that were lost by pointspread smearing and further averaging. The complicated pattern in the center of Figs. 4 and 5 has been remover and the analysis strongly suggests that the spoke tapering is continuing into the center. This result suggests to us that $P$ LOG $P$ maximum entropy analysis may be a useful technique for some image analysis problems. It will complement other methods.

It may be noted that the effect of grey edges of the pinhole could be included in the pointspread function. Also, if scattering in the image film is important, it may be included in the pointspread function. Further, the resilts are not restricted to dealing with photons. The transport of energy could just as well have been done tyy neutrons.

Further developmental work needs to be done on the method of solution of the nonlinear equations involved in this analysis. Currently we are using a form of Newton-Raphson procedure. It may be possible to find a more efficient method which is specific to the particular class of equation. It is also desirable to study the solution of the extremun condition which occurs in the analysis by the conjugate çradient method. If this could be done efficiently it would obviate the solution of the system of nonlinear equations.

\section{REFERENCES}

1. J. P. Burg, "Maximum Entropy Spectral Analys is," 37th Annual Meeting, Society of Exploration Geologists, Oklahoma City, October 1967, unpublished.

2. B. R. Frieden, "Restoring with Maximum Likelihood and Maximum Entropy," J. Opt. Soc. Am., 62, 821 (1972).

3. J. E. Brolley, R. B. Lazarus, and B. F. Suydam, "Maximum Entropy Restoration of Laser-Fusion Target X-Ray Photographs," Digest of Technical Papers, International Conference on Image Restoration and Analysis, U. of Toronto, Canada, July 1976.

4. H. J. Trusse11, "Some Notes on Linear Maximum a Posteriori Image Restoration," submitted for publication to IEEE Transactions on Acoustics, Speech, and Signal Processing, February 1977. 